International Journal of Modern Physics B

Vol. 31, No. 7 (2017) 1741014 (11 pages)

(C) The Author(s)

DOI: $10.1142 / \mathrm{S} 0217979217410144$

\title{
Power coupling characteristics between FBG and back-scattering signals
}

\author{
Jianzhi Li*, Desheng Zhao, Yuemin Hou and Baochen Sun \\ Key Laboratory of Structural Health Monitoring and Control, \\ Shijiazhuang Tiedao University, Shijiazhuang 050043, P. R. China \\ *lijianzhigang@163.com
}

Received 18 November 2016

Revised 26 December 2016

Accepted 25 January 2017

Published 28 February 2017

\begin{abstract}
The property and compatibility between fiber Bragg grating (FBG) and back-scattering signals are investigated by employing optical time domain reflectometry. We compare the power spectrums of spontaneous Brillouin scattering (SpBS), simultaneous Brillouin scattering (SBS) and Rayleigh scattering (RS), and coupling mechanism between FBG and back-scattering signal is explored. Experimental results show that the region of FBG contributes to the backscatter power and causes the desired reflection, and the power peak of FBG in SBS power spectrum is the sharpest among back-scattering light power spectrums and broadens with the decrease of spatial resolution. Moreover, the FBGbased method is used to find the location of temperature or stain event for scatter-based distributed sensors.
\end{abstract}

Keywords: Optical fiber optics; fiber distributed sensors; fiber gratings; scattering.

PACS number: 42.65.Es

\section{Introduction}

In recent years, distributed optical sensing techniques have been one of the most vibrant fields of research for health structural monitoring. ${ }^{-1} \underline{-3}$ Distributed sensing with meter and sub-meter spatial resolution along several tens of kilometers has been demonstrated. $\stackrel{4}{ }$ These include techniques based on Raman, stimulated Brillouin scattering (SBS), spontaneous Brillouin scattering (SpBS), and Rayleigh scattering (RS) as well as those involving multiplexed fiber Bragg gratings (FBGs). $\underline{4}-\underline{6}$

This is an Open Access article published by World Scientific Publishing Company. It is distributed under the terms of the Creative Commons Attribution 4.0 (CC-BY) License. Further distribution of this work is permitted, provided the original work is properly cited.

* Corresponding author. 


\section{J. Li et al.}

Techniques based on scatter typically employ optical time domain reflectometry. At the same time, technology combining BOTDA with FBG is becoming a brand-new and a highly interesting area. Technology of collinear temperature testing based on BOTDA and FBG sensing ${ }^{7}$ was proposed to test a large range of distributed temperature and local high-precision temperature. A highly-integrated hybrid sensing system that effectively combines the advantages of both standard BOTDA and in-line time-division-multiplexed (TDM) FBG-based sensing was proposed, $\stackrel{8}{,}$ allowing for simultaneous dynamic discrete strain measurements and distributed (static) strain/temperature sensing using a single optical fiber, a common pulsed narrowband optical source and a shared receiver unit. More recently, integrated optical fiber sensing system by combining large-scale distributed BOTDA/R and localized FBGs $^{9}-11$ was proposed for strain testing. The influence of wavelength of FBG on the Brillouin power spectrum was investigated in detail. 12 However, the effect of FBG on back-scattering signals has not still been investigated in these works. It is important to know the coupling mechanism and magnitude of each reflection for hybrid sensing system combining FBG and backscatter-based distributed sensor. Is the back-reflected signal from FBG beneficial or harmful to the backscatters? Until now, it is unclear about this information.

The goal of the work is to investigate the power coupling characteristics between FBG and back-scattering signal. Therefore, we explore influential mechanism of FBG on Brillouin signal as well as Rayleigh signal. Moreover, we compare the power spectrum of different back-scattering signal and infer with more confidence that FBG-based method is capable of being used as a positioning tool.

\section{Principles of Techniques Based on Scatter}

\subsection{FBG sensor}

An optical fiber can be perturbed by small changes of the refractive index. FBG is made of a series of refraction index changes, spaced at a constant length period, and used as a reflector which has great impact on the back-scatter power. Additionally, the period and refractive index of FBG region was changed by environmental temperature and strain, which alters the reflective wavelength of FBG given by the following equations:

$$
\lambda_{s}=2 n_{\mathrm{eff}} \Lambda
$$

where $\lambda_{s}$ is the refractive wavelength of FBG; $n_{\mathrm{eff}}$ is the effective index of FBG region; $\Lambda$ is the refractive index period. Therefore, we employ FBG as a reflector rather than a filter in this work.

\subsection{Rayleigh distributed sensor}

Rayleigh backscatter in optical fiber is resulted from the random fluctuations in the index profile along the fiber length. Variations in material density and compositional 
fluctuations occurring during fiber manufacture create the random inhomogeneities that give rise to the refractive index variations. For a given fiber, the scatter amplitude as a function of distance is random and static property of that fiber. This scatter amplitude can be modeled as a long and weak FBG with a random period. The scattered intensity is proportional to the changes in the local period of the Rayleigh scatter caused by an external stimulus (i.e., strain or temperature), and in turn causes the changes in the locally reflected spectrum. This spectral shift can then be calibrated to form a distributed strain or temperature sensor. Equation (2) shows a simple empirical relation for the RS loss and the wavelength in germanium-doped silica fibers:

$$
\alpha_{s}=\frac{(0.76+0.51 \Delta n)}{(\lambda / u m)^{4}}\left[\frac{d B}{k m}\right],
$$

where is the difference between the fiber core's refractive index and that of the fiber cladding; is the wavelength of light pulse into a fiber.

\subsection{Brillouin distributed sensor}

Brillouin backscatter includes SBS and SpBS. Brillouin scatter is induced by acoustic waves and shows a frequency shift given in Eq. (3) in the order of $10 \mathrm{GHz}$ which depends on the angle. The angle can be used to measure the scatters. This frequency shift is maximized in the backward direction. Different methods have been proposed for optical fiber sensors based on this effect. BOTDA and BOTDR are common among these Brillouin-based techniques. Brillouin frequency shift increases linearly with (longitudinal) strain and temperature [see Eq. (4)].

$$
\begin{aligned}
& \nu_{B}=\frac{2 n V_{a}}{\lambda_{0}}, \\
& \nu_{B}=\nu_{B}(T, 0)\left(1+4.48 \Delta \varepsilon+1.18 \times 10^{-4} \Delta T\right),
\end{aligned}
$$

where $n$ is the fiber core's refractive index, $\lambda_{0}$ is the wavelength of light pulse into a fiber, $V_{a}$ is the velocity of acoustic waves in optical fiber, $\nu_{s}$ is Brillouin frequency shift, $\Delta \varepsilon$ is the strain variation and $\Delta T$ is the temperature variation.

As a result of these attenuation effects, light traveling along a fiber exhibits an exponentially decreasing power level with the distance. The power transmission relation between incident light $P_{0}$ and transmitted power $P(z)$ at a distance $z$ is expressed as

$$
P(z)=P_{0} \cdot e^{-\alpha z}
$$

Let us now focus on the effect of scattering and consider a laser pulse in a fiber with the temporal pulse duration $\tau$. As the pulse propagates, light is scattered within a fiber element of length $W$, as depicted in Fig. $1 . W$ is given as

$$
W=\tau \cdot v_{\mathrm{gr}}=\tau \cdot \frac{c}{n_{\mathrm{gr}}}
$$




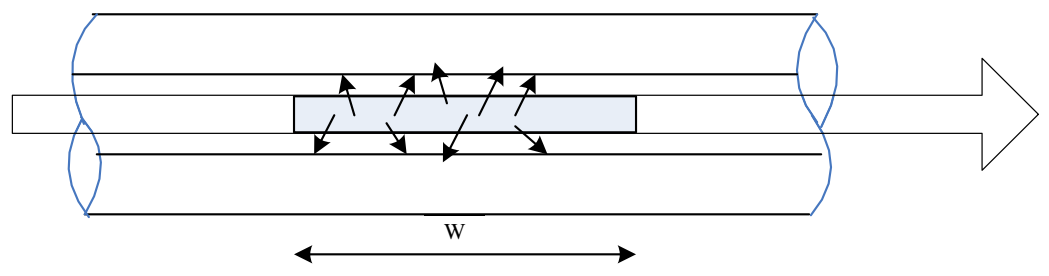

Fig. 1. Scattering within a fiber element of width $W$.

where $v_{\text {gr }}$ is the group velocity, $c$ is the speed of light in vacuum, and $n_{\text {gr }}$ is the group index of glass.

The scattered power $d p_{s}$ at the position $z$ within an infinitesimal small interval $d z$ is proportional to the pulse power $P(z)$.

$$
\begin{gathered}
d p_{s}=k \cdot P(z) d z \\
k=S \cdot \alpha_{S} .
\end{gathered}
$$

$S$ is the fraction of the light scattered in all directions that is captured by the fiber core, $\alpha_{s}$ is the scattering coefficient.

Summing up the light power backscattered from infinitesimal short intervals $d z$ from the whole pulse and taking the fiber attenuation into account, one can obtain

$$
P_{s}(L)=\int_{0}^{W} S \cdot \alpha_{S} \cdot P_{0} \cdot \exp \left(-2 \alpha\left(L+\frac{z}{2}\right)\right) d z .
$$

Likewise, for short pulse width, the product of $\alpha$ and $W$, Eq. (9) can be simplified to

$$
P_{s}(L)=S \cdot \alpha_{S} \cdot W \cdot P_{0} \cdot e^{-2 \alpha L}
$$

Equations (9) and (10) shows the fact that for narrow pulse, the backscattered power is proportional to the pulse duration $\tau$.

\section{Experiment and Material}

In this work, the main objective of a Neubrex NBX-7020 measurement is to determine power coupling characteristics between FBG and back-scattering signal. Three samples with FBGs written along the Corning 28E optical fiber were used

Table 1. FBG parameters in Corning 28E optical fiber.

\begin{tabular}{clllcr}
\hline Sample & Wavelength/nm & Reflectivity/\% & Bandwidth/nm & $\begin{array}{c}\text { Distance } \\
\text { between FBGs }\end{array}$ & $\begin{array}{c}\text { Length of } \\
\text { tail fiber } / \mathrm{m}\end{array}$ \\
\hline 1 & 1459.82 & 92.055 & 0.20 & - & $7.90 \mathrm{~m}$ \\
2 & 1499.95 & 88.548 & 0.22 & - & $8.00 \mathrm{~m}$ \\
3 & $1514.80,1519.79$, & $90.98,90.68$, & $0.22,0.23,0.22$ & $8.02,8.01$ & - \\
& 1524.8 & 89.50 & & & \\
\hline
\end{tabular}



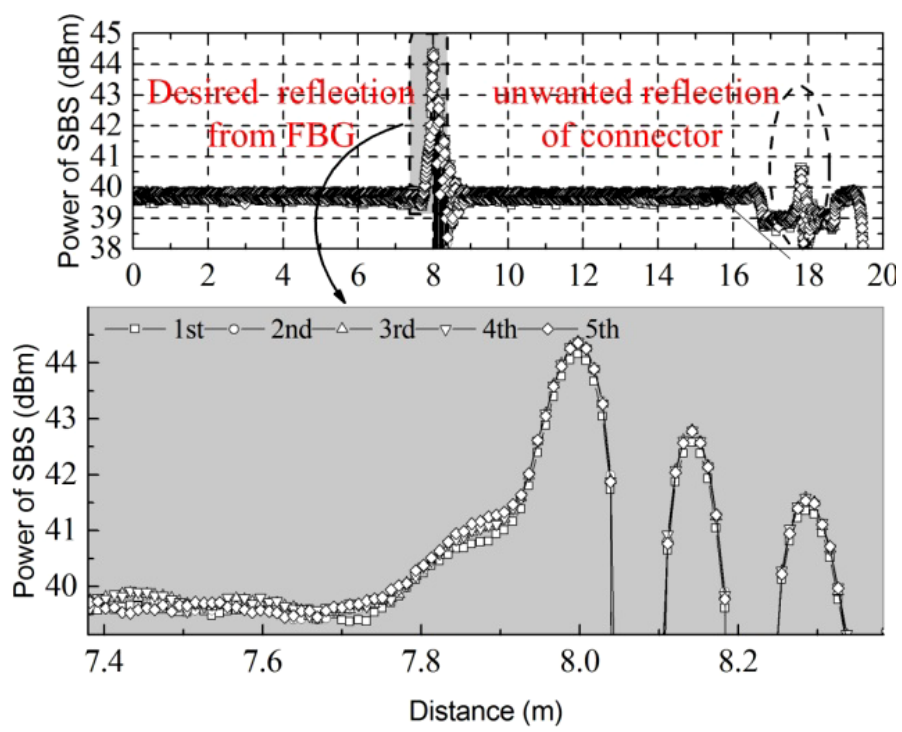

Fig. 2. Power spectrum of SBS along the fiber with $2 \mathrm{~cm}$ spatial resolution for sample 1.

to measure RS, SpBS, SBS power spectrum to investigate the power coupling characteristics between FBG and back-scattering signal. FBG parameters are listed in Table 1. FBG points with different wavelengths have been written along the sensing fiber, the so-called in-line FBGs fiber. Figure 2 shows a measurement setup with a distributed sensing instrument and a segment of in-line FBGs sensing fiber. The low and high wavelength ends of in-line FBGs fiber were, respectively, connected with the "pump" connector and the "probe" connector of optical instrument. Neubrex NBX-7020 was employed in all the tests presented in this paper. The backscatter signal is a function of pulse width, fiber-length, and fiber parameters. Therefore, the scatter profile is measured over length of up to $8 \mathrm{~m}$ (specimen $1 \#$ and $2 \#$ ) or $16 \mathrm{~m}$ (specimen $3 \#$ ) with $2 \mathrm{~cm}$ (corresponding to $0.2 \mathrm{~ns}$ pump pulse width), $5 \mathrm{~cm}$ (corresponding to $0.5 \mathrm{~ns}$ pump pulse width), $10 \mathrm{~cm}$ (corresponding to $1 \mathrm{~ns}$ pump pulse width), $20 \mathrm{~cm}$ (corresponding to $2 \mathrm{~ns}$ pump pulse width), $50 \mathrm{~cm}$ (corresponding to $5 \mathrm{~ns}$ pump pulse width) and $100 \mathrm{~cm}$ (corresponding to $10 \mathrm{~ns}$ pump pulse width) spatial resolution by RS and SBS measurement. Due to the ability of the NBX-7020 instrument, the minimum spatial resolution of SpBS measurement is set as $20 \mathrm{~cm}(2 \mathrm{~ns})$. The sampling rate is $1 \mathrm{~cm}$ and the optical refractive index is set as 1.46. All measurements were done in the room temperature $\left(25^{\circ} \mathrm{C}\right)$. Techniques based on backscatters typically employ the optical time domain reflectometry.

\section{Results and Discussion}

Figures 2-5 illustrate a typical SBS, SpBS, RS measurement display. At a glance, reflection peaks in the site of FBG can be seen because of reflections from FBG. This 


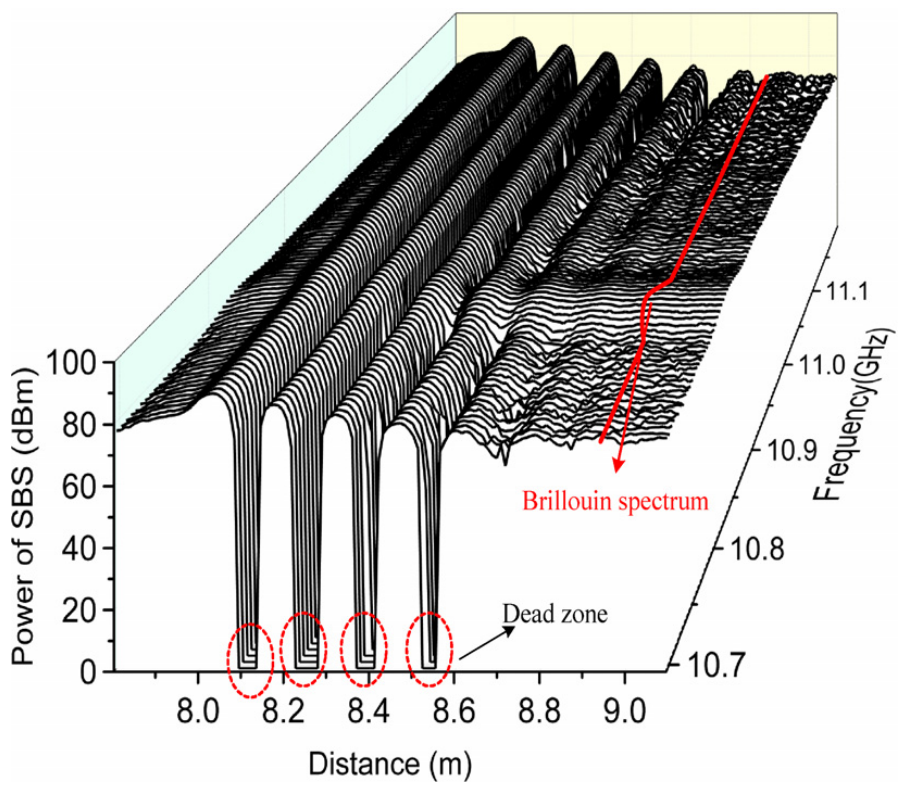

Fig. 3. Brillouin gain spectrum of $1 \#$ sample.
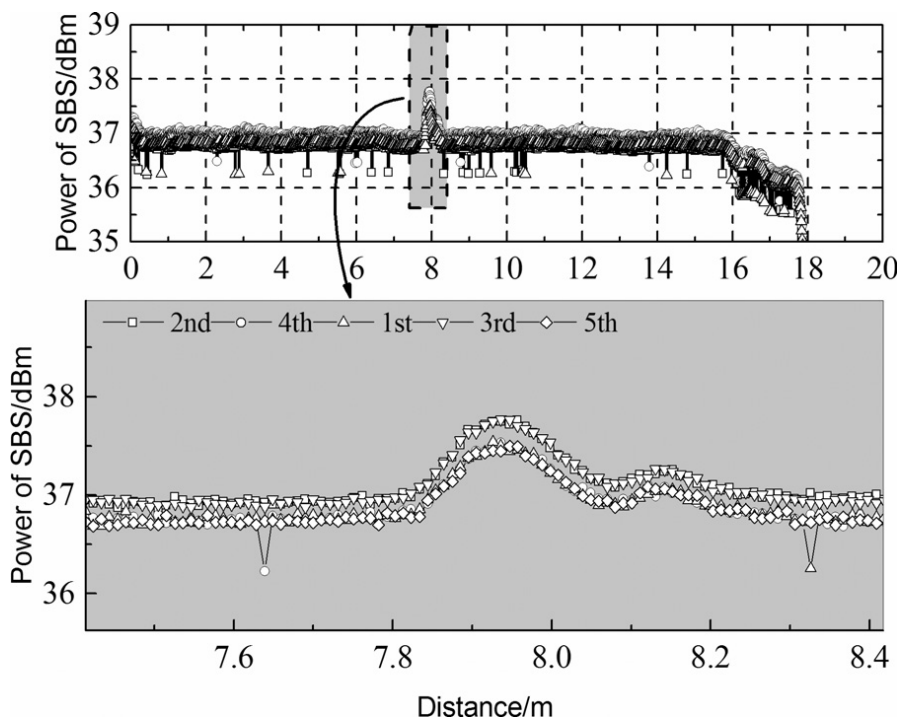

Fig. 4. Power spectrum of SpBS along the fiber with $2 \mathrm{~cm}$ spatial resolution for sample $1 \#$.

is useful information for a positioning design. The roof of the peaks indicates that the shape is the sum of the reflected signal from FBG (due to FBG filter) and the backscatter signal. This reflection event could be used to map an external stimulus, such as strain or temperature. The reflection peak profile of backscatters is found 

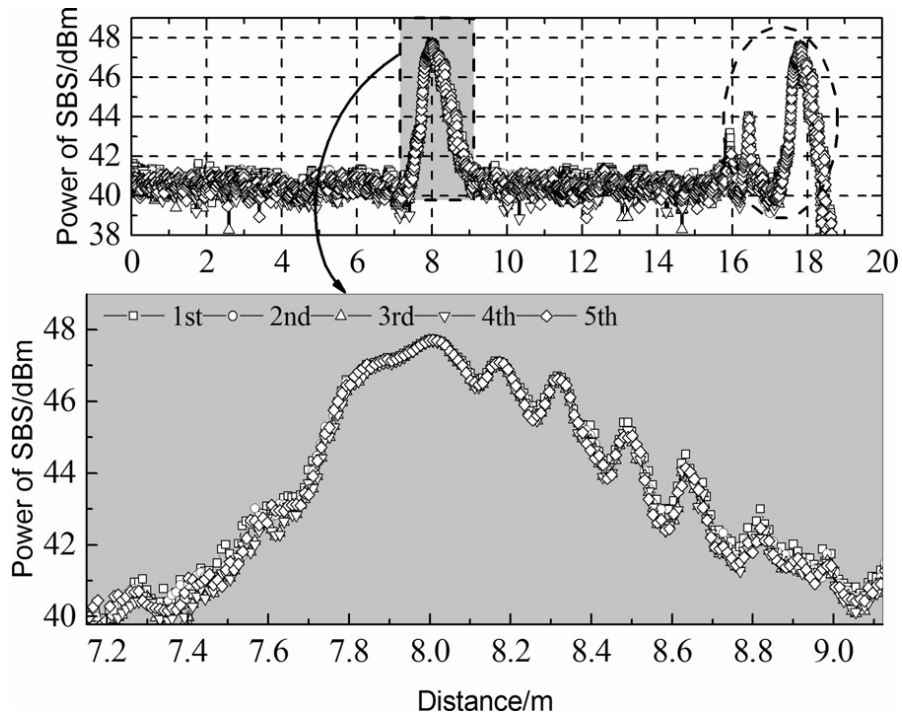

Fig. 5. Power spectrum of RS along the fiber with $2 \mathrm{~cm}$ spatial resolution for sample $1 \#$.

to be different in the tests. The sharpest spectrum of SBS (0.2 ns pulse, as shown Fig. 2) is considered as a favorable positioning tool. Optical power at FBG point is reflected and superimposed with back-scattered light, the power level of which is a function of the chosen pulse width. Therefore, the actual height seen on the Neubrex NBX-7020 display depends on pulse width for a given in-line FBGs fiber. With pump pulse width increasing, the reflected power peak shape is broadened gradually in SBS measurement, and the height of the reflection becomes lower and lower, as depicted in Fig. 6. Compared with the reflection magnitude in SBS measurement, the $2 \mathrm{~ns}$ ( $20 \mathrm{~cm}$ spatial resolution) pulse tends to broaden the reflected peak in SpBS measurement. However, why is the reflected power peak wider compared to sharp power peak of SBS when the same pulse is used in RS measurement? It could be considered that the sharp shape is caused by the SBS signal filtered at the window of Brillouin frequency, and the shape and magnitude of RS cover entire pump light wavelength range.

Dead zones are always related to the presence of reflections. In our tests, dead zones occur when the reflected signal from FBG saturates the optical receiver of NBX-7020. Dead zones (approximately $1 \mathrm{~m}$ ) occurred in SBS and RS measurement (as illustrated in Figs. 3 and 5). The receiver is slow to recover its sensitivity after the saturation resulting in the loss of information. If the receiver saturates because of strong signals, it will take some time to recover from this overload condition. As a consequence, the measured fiber response is superimposed by the receiver's overload behavior, yielding a distance fiber segment (from 8 to $9 \mathrm{~m}$, as depicted in Figs. 3 and 5) covered by a diminishing tail. Dead zone has great impact on SBS and $\overline{\mathrm{RS}}$ signal, which leads to the error of strain or temperature. The attenuation 
Table 2. Comparison of positioning accuracy for sample $1 \#$.

\begin{tabular}{lc}
\hline Testing methods & Average positioning/(repeat five times) \\
\hline BOTDA & 8.01 \\
BOTDR & 7.936 \\
COTDR & 8.008 \\
Actual length & 7.90 \\
\hline
\end{tabular}
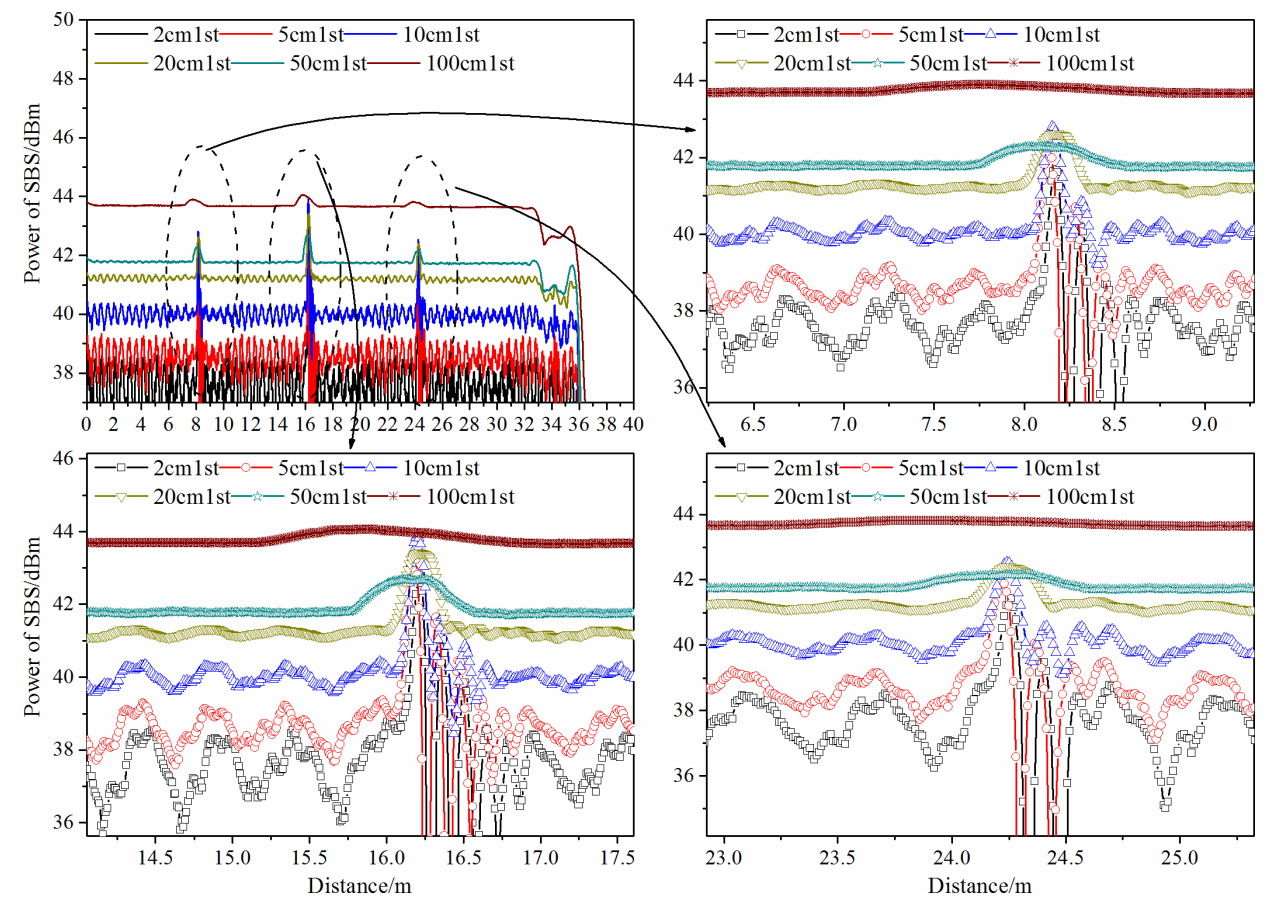

Fig. 6. Power spectrum of SBS along the fiber with different spatial resolutions for sample 3.

dead zone is defined as the distance from the start of the reflection peak point to the point where the receiver has recovered to the settled backscatter trace. The effects due to dead zone can be solved by exchanging both optical fiber ends to attain tail information. Then, for more security, we should set the interval distance between FBGs as more than $1.5 \mathrm{~m}$ in applications.

Figure 2 illustrates the power spectrum of SBS along the fiber for sample 1\# with $2 \mathrm{~cm}$ spatial resolution. The reflected peak (approximately $18 \mathrm{~m}$ ) located in the broken ellipse is the unwanted reflection from optical connector set in the instrument because the length is more than $15.8 \mathrm{~m}$ actual length. Additionally, it is also shown that the power has a good repeatability in Fig. 2.

From Table 2, a slight positioning difference in RS, SpBS, SBS power spectrum for sample $1 \#$ can be seen, compared to the actual length from ruler. The lengths 


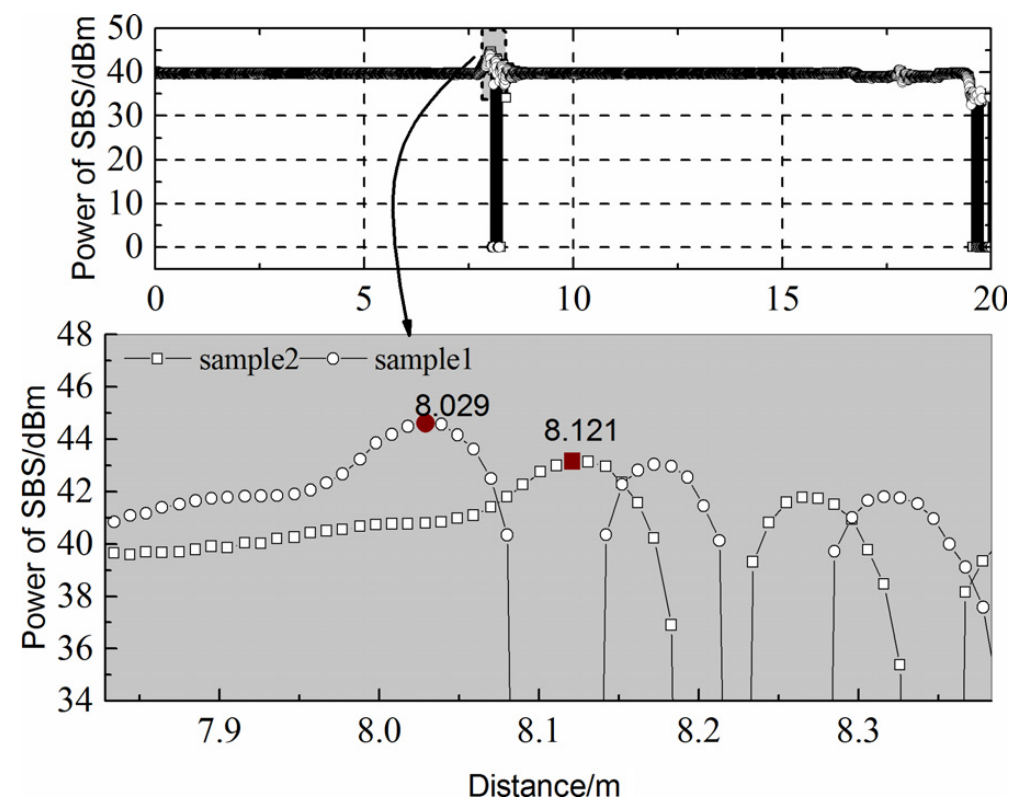

Fig. 7. Power spectrum of SBS along the fiber with $2 \mathrm{~cm}$ spatial resolution for sample $1 \#$ and $2 \#$.

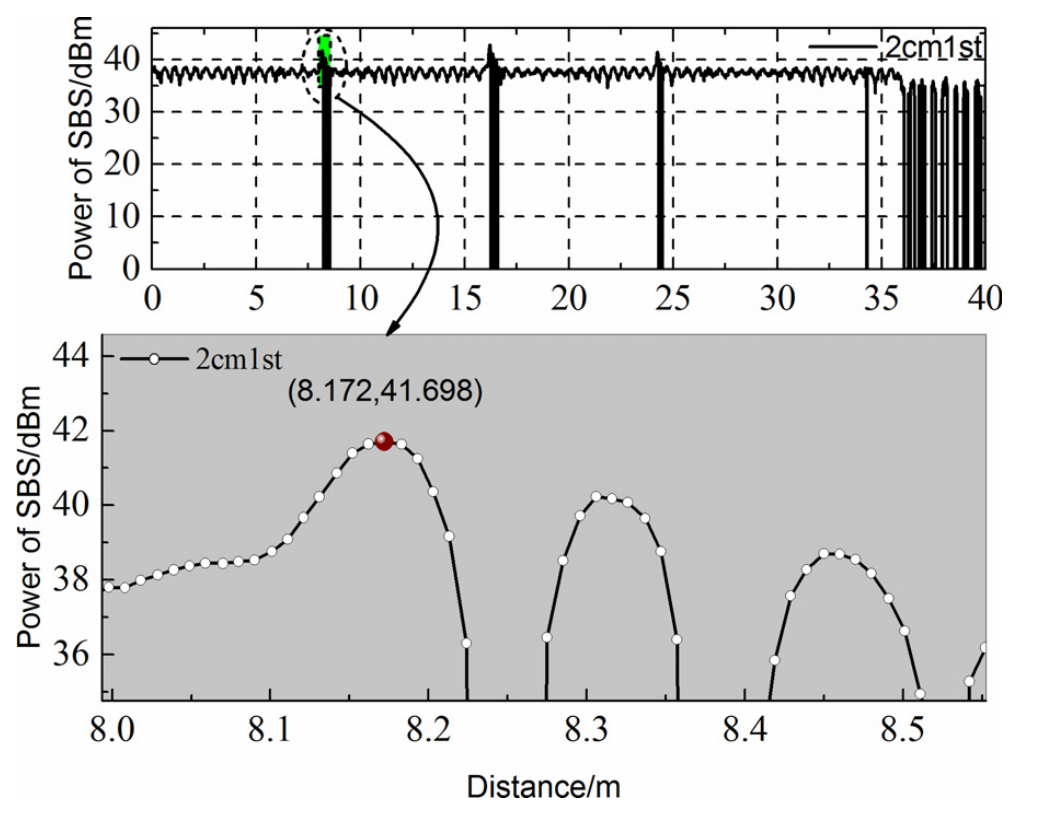

(a)

Fig. 8. Power spectrum of SBS along the fiber with $2 \mathrm{~cm}$ spatial resolution for sample 3 . (a) Enlarged view of peak 1; (b) enlarged view of peak 2; (c) enlarged view of peak 3. 

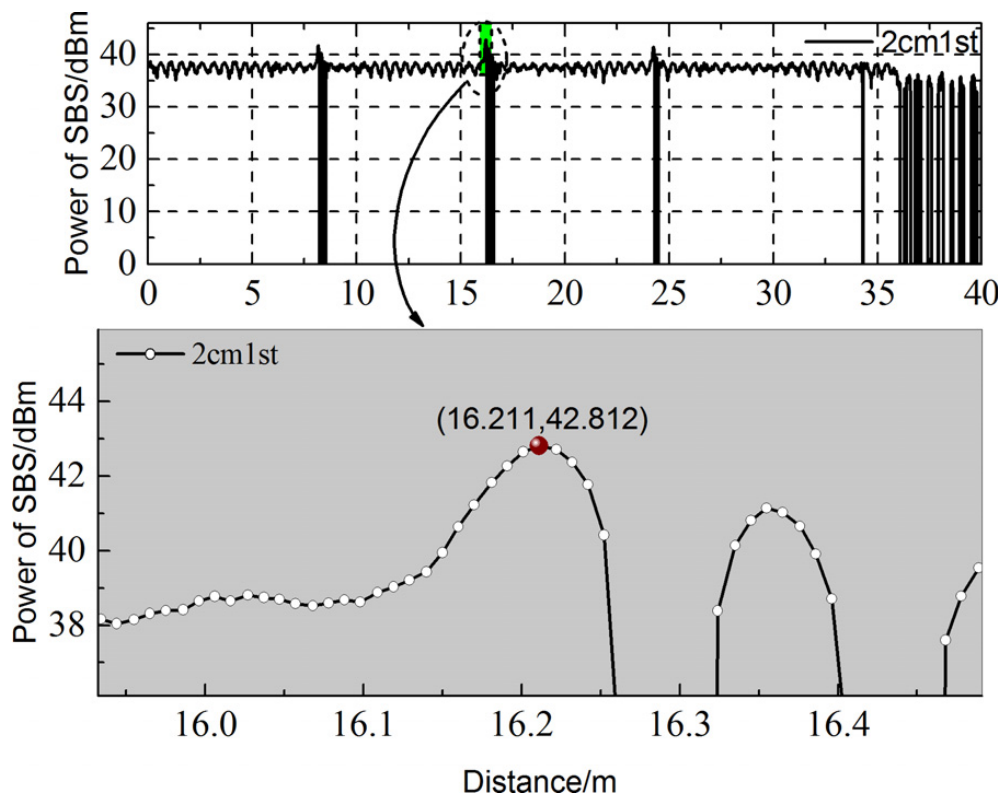

(b)
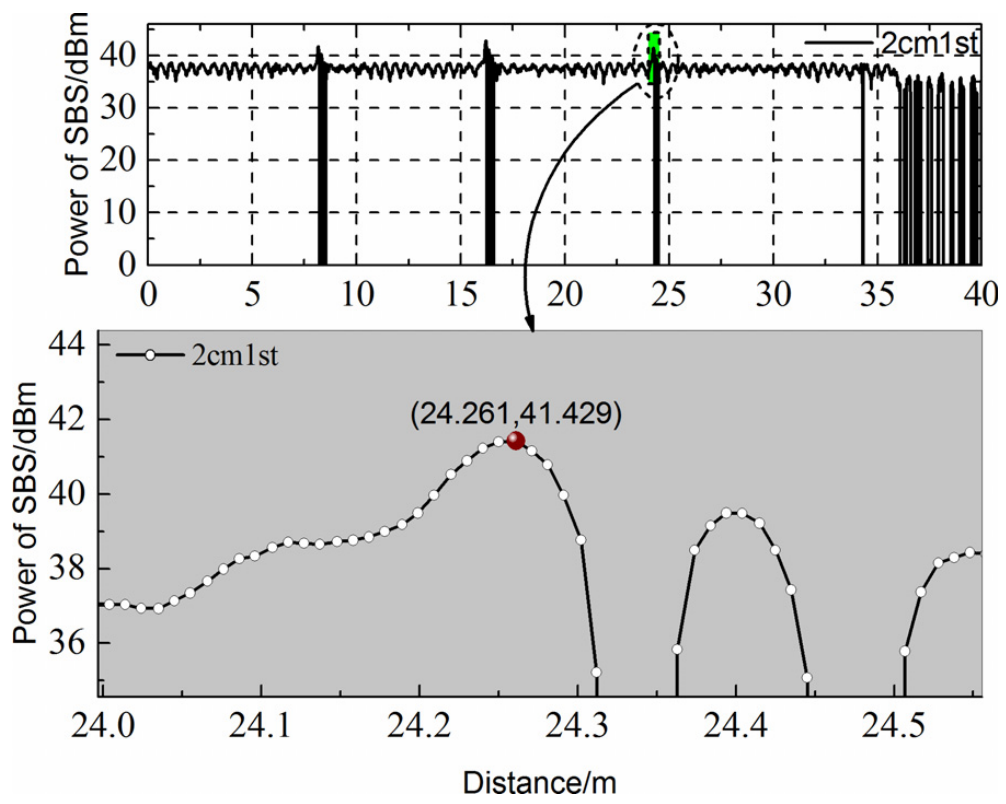

(c)

Fig. 8. (Continued) 
from NBX-7020 are 8.01, 7.936 and $8.008 \mathrm{~m}$, respectively. Compared with the actual length of $7.90 \mathrm{~m}$, the absolute positioning error depends on the properties of the NBX-7020 instrument. The difference in length between samples $1 \#$ and $2 \#$ is really $10 \mathrm{~cm}$, which is in agreement with the length attained by NBX-7020 instrument (as displayed in Fig. 7, 8.029, and $8.121 \mathrm{~m}$ ). Moreover, it is shown from Fig. 8 that the interval between $\overline{F B G s}$ for sample $3 \#$ is 8.039 and $8.05 \mathrm{~m}$, which matches with the actual distance $(8.02,8.01 \mathrm{~m})$. Consequently, the FBG-based positioning method provides a very feasible and practical means for accurate positioning of distributed sensors.

\section{Conclusions}

In this work, we explore coupling mechanism of FBG on Brillouin signal as well as Rayleigh signal. It is found that the region of FBG contributes to the magnitude of backscatters and causes the desired reflection. The sharp power of SBS with $2 \mathrm{~cm}$ spatial resolution is an accurate positioning method. Dead zones occur when the reflected signal saturates the receiver, which can be solved by exchanging both optical fiber ends to attain tail information. Therefore, for more security, the interval between FBGs should be set as more than $1.5 \mathrm{~m}$ in engineering applications. Moreover, the power spectrums of backscatters have a good repeatability. The FBG-based positioning method is feasible and practical.

\section{Acknowledgments}

The support of National Natural Science Foundation of China (No. 51508349), Department of Human Resources and Social Security in Hebei (CL201626, A2016002035) and the Natural Science Foundation of Hebei Province (No. E2015210094) are acknowledged.

\section{References}

1. A. Farhad, J. Intell. Mater. Syst. Struct. 18, 879 (2007).

2. X. Feng et al., J. Eng. Mech.-ASCE 139, 1797 (2013).

3. X. Feng et al., J. Eng. Mech. 140, 1 (2014).

4. K. Kishida, Y. Yamauchi and A. Guzik, Photonic Sensors 4, 1 (2014).

5. K. Nishiguchi et al., Sensors 14, 4731 (2014).

6. H. Feng et al., Chin. Opt. Lett. 11, 0206071 (2013).

7. T. Nannipieri et al., in Proc. 22nd Int. Conf. Optical Fiber Sensors (Beijing, China, 2012), pp. 1-4.

8. J. Zhang et al., Optical Technique 39, 247 (2013).

9. Z. Zhou, J. He and J. Ou, Measurement 47, 698 (2014).

10. C. Lan, H. Liu and Z. Zhou, China. Civil. Eng. J. 46, 55 (2013).

11. F. Zaidi et al., OFS2012 22nd International Conference on Optical Fiber Sensors, Applied Optics 51, 7268 (2012).

12. J. Li, B. Sun and K. Kinzo, J. Civ. Struct. Health Monit. 5, 629 (2015). 